I 965 International conterence

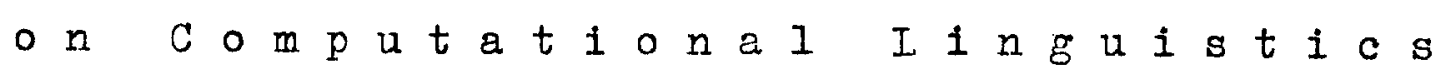
$S O M E Q U E S T I O N S \quad O F$ I A N G UAG T H $\mathbb{D} O \mathrm{R} Y$

S. A b r a h a m

53, Uri u., Budapest I., Hungary

Computing Centre of the Hungarian Academy of Sciences

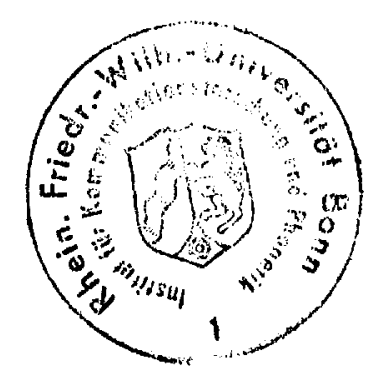


ABSTRACT. It is shown that the assumption that language is non-finite irvolves the use of a constructive logic which leacs to some restrictions on language theory and to the fact that the only possivie definition of language is that proposed by generative grammars. Generative grammars can be formulater as normal Markov/ alcorithms anc thus their study can be reducer to the stury of such algorithms of a special tyne. I new type of renerative grammar is defined, called matrix grammar. It is shown that a language generated by a context-restricted grammar can be also eenerated by a matrix srammar. Some properties of matrix grammars are shown to be cecicable. The problem of the explicative power of generative Erammars is riscussed.

1. Ianguage metatheory, as irdeed any metatheory, must exactly specify the operations allowed in building up the theory /of language/. This may be cone by choosing the loric of the theory. If Ianguage is consideres a non-finite set, a constructive logic /Kolmogorov/ must be choosen. This entails some restrictions on the notions and methods to be used ir language theory. Namely, we can not speak of actually infinite sets, and we can not use the quantifiers "there exists" anc "all". Thus we can not include in language theory the notion of 'language' itself in the usual way, as the set of all /grammatically or semantically/ correct sentencos. Similarly, we can not make use of "distributional analysis" /at least without any restrictinns/, as it generally has the form: 
/the sentence/ $s_{1}$ has the property $R_{1}$, if there exists / a sentence/ $s_{2}$ with the property $\mathrm{R}_{2}$ /not necessarily $\mathrm{R}_{1} \neq \mathrm{R}_{2} /$.

It follows that the single way of defining language is that proposed by generative grammars. These grammars are in fact devices that produce /gererate/ the sentences of a language /and only those/, one after the other. So, at every moment we have generated a finite set of sentences, and at the same, if the grammar is properly constructed, at evry moment we can cenerate a sentence not yet generated before. So, in fact the language/the set of all the sentences of the language/ is a potentially infinite set and the abovementioned difficulties do not arise. The restrictions to be respected within generative grammars as to the /logically correct/ notions and operations are precisely formulated /it. may be interesting to note that Chomsky coes not respect all. of them/.

2. Most of the properties /and possibly even the most important ones/ of generative grammars are obtainer by constructing automata, equivalent to 
Abraham 4

different generative grammars, and in this way using the results of automata theory. It is shown that a more natural /anc easy/ way to study generative grammars is to formulate them as normal /Markov/ algorithms [7], [1]. so, if given a Phrase Structure Grammar $G$ it can be given a finite set of normal algorithms $\bar{G}=\left\{e x_{i}\right\}$ so that by aplying the algorithms to the initial strings we obtain the language generated by $G$.

The algorithms $e r_{i}$ have the properties:

(i) each rule /of the algorithm/ rewrites at once only one symbol;

(ii) by applying a rule to a string the length of the string is not diminished.

For constructing $\vec{G}$ we must be able to compose the normal algorithms so that these pronerties should be rreserved. The composition rule given by fiarkov does not fulfll this condition. So the following composition rule is proved and used:

If $e r_{v_{p}}, \mathcal{L}_{v_{p}}$ are two normal algorithms with the properties ( $i$ ) and (ii) then for every $\sigma \in \Sigma$ /the set of Intial strings/ we have

$$
\mathcal{L}(\sigma)=(\operatorname{er} \circ \mathcal{L})(\sigma)=\mathcal{L}(\operatorname{er}(\sigma))
$$


where $\mathcal{L}$ is a normal algorithm with the scheme

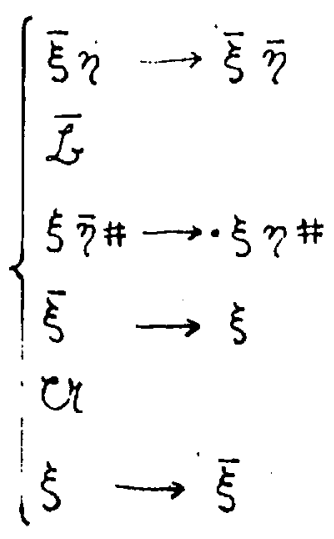

where $\xi, \eta \in V_{p} ; \bar{\xi}, \bar{\eta}$ are symbols put in one-to-one correspondence to the symbols from $V_{p}$ land different from them and between them/; $\vec{E}$ is the list of the rules of the algorithm \& with every $\xi$ changed to $\dot{\xi}$. Evidently $\mathcal{L}$ has the proferties (i) and (ii).

It is shown that to a set of algorithms $\bar{G}=\left\{e r_{i}\right\}$ a single algorithm $e r$ corresponds if $\Sigma /$ the set of the initial strings/ is proferly enlarged, so that $I_{(}(G)=e r(\Sigma)$. Thus the study of ESG is reducible to the study of normal algorithms of the tyne of $U$ / the rewriting rules of which axe, in fact, context-restricted rules/. The sufficient and necessary conditions are estarlisher for generating a non-finite language /hy different generative grammars/. 
It is shown that each singular transformation /Chomsky/ can be formulated as an algorithm of type er.

The most studied generative grammars are the context-free grammars /CFG/ and the context-restricted grammars /CRG/. Some properties of these grammars are consicered to be undecidable. In tris respect they are also different. The differences are formulated in Table 1 [6]:

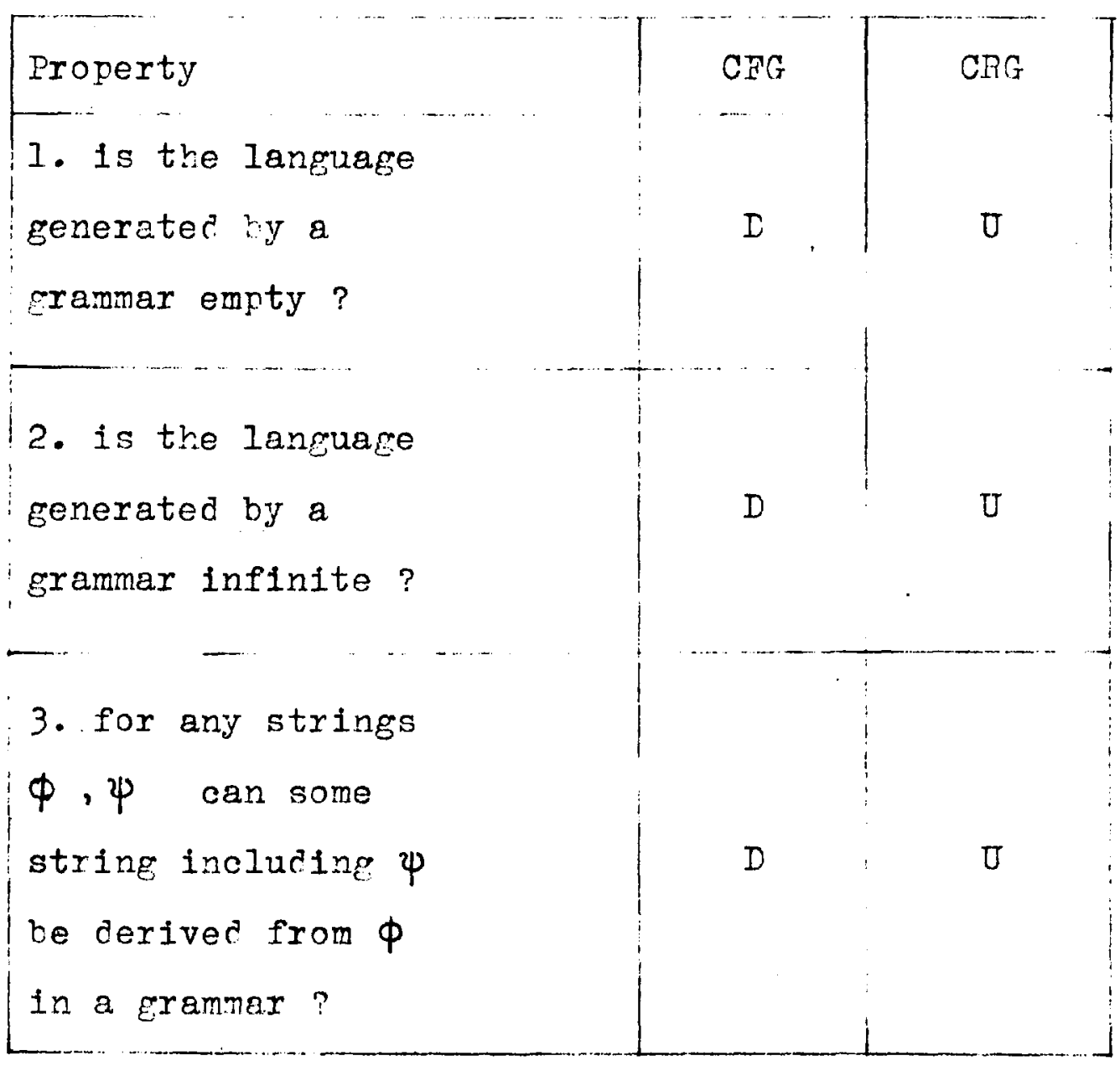




\section{Abraham 7}

where $D$ indicates that the property in question Is decidable, $U$ that it is undecidable.

The CF grammars have not the necessary generative power to model natural languages. The CR grammaxs may have this power /altough this froblem has not been cleared up/ but the undecibality of the properties 1 - 3 /especlally, 3/ makes highly doubtful their fitness for moreling natural languages.

A new type of generative grammars is proposed under the name of matrix grammars /MG/ [2].

A matrix grammar is a quintuple

$$
G=\left(V, V_{t}, \Sigma, F, F^{*}\right)
$$

where

$$
\bar{G}=\left(V, V_{t}, \Sigma, \bar{T}\right)
$$

is a context-free frammar and $F^{*}$ is a finite set of matrices /called matrix rules/ defined as follows:

(1) $f^{*}$. is a matrix rule if it has the form

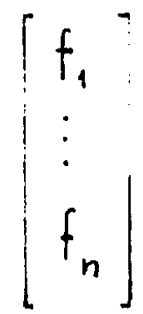

$f_{i} \in F \quad(1 \leq i \leq n)$ and not necessarily $\quad f_{i} \neq f_{j}$; 
(2) $f^{*}$ is a matrix rule if it has the form

$$
\left[\begin{array}{l}
f_{1}{ }^{*} \\
\vdots \\
f_{n}
\end{array}\right]
$$

where $f_{i}^{*}$ are matrix rules or helone to $F$.

To apply a matrix rule $f^{*}$ to a string $x$ means to aprly to $x$ all the context-free rules which form it, in the glven order /to annly a $S F$ rule to a string means to replace the first occurence of its left-side with its right-side/. If at least one of these context-free rules can not be applied to $x$, we say that $f^{*}$ can not he apolied to $x$.

It is shown that for any context-restricted Eramar $G$ it 1 s possible to construct a/stronmly/ equivalont matrix grammar.

For instance, the /not context-free/ language

$$
I:=\left\{a^{n} b^{n} c^{n}\right\}
$$

is generated by the matrix grammar

$$
G=\left(V, \nabla_{t}, \Sigma, F\right)
$$

with

$$
V=\{S, X, Y, Z, a, b, c\} ; V_{t}=\{a, b, c\} ; \Sigma=\{S\}
$$




$$
\begin{aligned}
& F:[S \rightarrow a b c] {\left[\begin{array}{l}
S \rightarrow a X b Y c Z] \\
Y \rightarrow a X \\
Z \rightarrow b Y
\end{array}\right] } \\
& {\left[\begin{array}{l}
X \rightarrow a \\
Y \rightarrow b \\
Z \rightarrow c
\end{array}\right] }
\end{aligned}
$$

It is shown that the properties $1,2,3$ are decicable for matrix grammars. So the statement that they are undectiable for t!e Ch grammars is erroneous the exroneousness of the rroof of the undecidability of property 3 given in [5] can be easily shown/. So the fitness of these grammars for modeling natural languages 1s most likely.

As we have mentioned, for each singular transformation a normal algorithm can be constructed which contains anly context-restricted rules. Leparting from this, it can be skown trat for a transformationel grammar /containing only singular transformations, see [4] / a weakly equivalent matrix grammar can be constructed. 
The matrix grammars can be formulated as a normal algorithm, too.

Since any normal / Markov/ algorithm can he reverser, it is possible to devise a method for the construction of a recognition grammar corresfonding to any given generative grammar. is the matrix grammar corresponding to a transformational grammar is, in general, only weakly equivalent to the latter, and in automatic /natural/ language processing /and especially in machine translation/ the adequate analysis is a crucial requirement, the to strong requirement of Chomsky to cerive the structure of a generatec sentence from the why it is generater, is drorrec, anc the matrix erammar is completed with a definitional apparatus /IA/ that makes it possible to assign to a generated sentence the same structure /analysis/ as is assigned by a transformational grammar /details see in [3] /. By constructing the recognition grammar corresponding to a given cenerative grammar, the DA of the Eenerative crammar is taken over. 
3. Some examples are shown how the above consicerations can re applier to automatic proceseing of natural lancuages.

\section{BIBIIOGRAPHY}

[I] Abraham, s., A Formal Stucy of Generative Grammars I, Computational Iinguistics II, pp. 5-18, 1964

[2] Abraham, S., Some Questions of Phrase Structure Grammars I,/under press in Computational Inguistics IV/

[3] Abraham, S., Some questions of Ianguage Theory Kiefer,F., /undex press in Acta Hungarica/

[4] Thomsky, i., Categories and syntactic Theory, MIT Press, 1964

[5] Chomsky, $N_{0}$, Formal rroperties of Grammars in Hancbook of rathematical Esychology, J.Hley and Sons, Inc. New York, NY, vol.2, $r p \cdot 323-418,1963$

[6] Iandweber,F.S., Lecision Eroblems of Phrase Structure Grammars, IFE Trans.,vol.EC-13, pp.354-362, 1964

[7] Markov,A.A., Teori,ja algorifmov, Trudy Mat. Inst. AN SSSR, roscow, 1954 